\title{
Effects of Flexible Pole Training Combined with Lumbar Stabilization on Trunk Muscles Activation in Healthy Adults
}

\author{
Jae-Heon Lim \\ The Lab Cooperative 31, Baekseo-ro 137beon-gil, Dong-gu, Gwangju, Korea.
}

Objective: This study aimed to determine the efficacy of flexible pole training combined with lumbar stabilization in improving trunk muscle activities and to investigate the difference according to posture in young adults.

Methods: Twenty-five participants were enrolled in this study. The subjects were randomly allocated into either the flexible pole group or the rigid pole group. Participants performed lumbar stabilization exercises on quadruped and curl-up, with the flexible pole or rigid pole. Electromyography was used to assess the percent maximal voluntary isometric contracion (\%MVIC) of the rectus abdominis (RA), external oblique (EO), internal oblique (IO), and erector spine (ES) muscles. All participants completed one 30-minute session per day, 3 days per week, for 6 weeks. The evaluation was performed before and 6 weeks after the training, and follow-up. The data were analyzed using independent t-test and two-way repeated measure analysis of variance to determine the statistical significance.

Results: The flexible pole in curl-up showed significant differences in EO and IO muscle activities compared with the rigid pole. The flexible pole in quadruped showed significant differences in 10 and ES muscle activities compared with the rigid pole. The RA, EO, IO, and ES muscle activities of both groups were significantly higher after 6 weeks training.

Conclusion: The flexible pole in curl-up and quadruped showed an improvement in trunk muscle activation. The flexible pole combined with lumbar stabilization will be useful as an exercise tool to improve activity of trunk muscles.

Keywords: Flexible pole training, Trunk muscle activation, Lumbar stabilization, Electromyography

\section{INTRODUCTION}

Lumbar spine stability is maintained through the interconnected actions of active (muscles), passive (bones), and neural systems. ${ }^{1}$ Stability of the trunk depends on the coordinated activity of many trunk muscles. The front, back, and side muscles of the spine cooperate to ensure the stability in various load conditions. ${ }^{2}$ The stable production and strong contractility in instantaneous position and velocity imposed on the spine are based on trunk stability. ${ }^{3}$ Stabilization exercise can activate more trunk muscle concurrently, and the load should be applied to the appropriate load specifically on the spine. Stabilizing the muscle groups of the body integrates global, and local muscles. ${ }^{4}$ The global muscles include the rectus abdominis (RA), external oblique (EO), and internal oblique (IO). The local muscles includes more deep trunk muscles such as the transverse

Received Nov 8, 2017 Revised Jan 16, 2018

Accepted Jan 24, 2018

Corresponding author Jae-Heon Lim

E-mail limjaeheon@gmail.com abdominis (TrA), multifidus (MF), and interspinalis. The small muscles are effective in controlling the stiffness of the spinal segment and in maintaining the posture of the spine. ${ }^{1}$

Trunk stabilization exercises are considered to be important. ${ }^{5}$ As a part of trunk stabilization training, swiss ball exercises on an unstable surface, ${ }^{6}$ core exercises, ${ }^{7}$ curl-up exercise, ${ }^{8}$ and the Pilates exercise $^{9}$ has been introduced in the literature. Although there have been several randomized controlled trials on the usefulness of traditional trunk exercises, ${ }^{10}$ selective training of stabilizing trunk muscles has been increasingly gaining attention. In recent years, flexible pole exercises using vibration stimulation have commonly have been used to improve body strength and improve coordination and balance skills in various fitness centers and rehabilitation institutions. ${ }^{11,12}$

Muscles can be strengthen when the neuromuscular stimulation

Copylight (C2018 The Korea Society of Physical Therapy

This is an Open Access article distribute under the terms of the Creative Commons Attribution Non-commercial License (Http:// creativecommons.org/license/by-nc/4.o.) which permits unrestricted non-commercial use, distribution, and reproduction in any medium, provided the original work is properly cited. 
or various feedbacks are applied. Vibration may help strengthen muscle strength. Vibrations applied to connective tissues can be used for treatment of patients by helping to improve the proprioception and motion perception. ${ }^{13}$ During muscle contraction, the vibration helps improve muscle strength. Lumbar stabilization involves more deep muscles through the movement of the limbs as well as the trunk. Active vibration contributes to muscle strength during pulling dynamic contraction. ${ }^{14}$

Flexible pole is designed to weigh the elastic characteristics and move of small amplitude of $5 \mathrm{~Hz}$ which is transmitted vibrations to the whole body enable continue to physically stable state. ${ }^{15}$ Trunk stabilizing muscles is stimulated through the lower amplitude vibrations, which are easily applicable and secure. ${ }^{16}$ Vibration occurring in the flexible pole is advantageous in that users can self-adjust the rate and amplitude of the active vibration stimulus caused by itself ${ }^{17}$ and safety in a variety of postures. ${ }^{18}$ It is also commonly used for upper extremity stabilization by performing bending, twisting or oscillating movements using a flexible pole.

Mileva et al. ${ }^{15}$ reported that flexible pole with vibration showed significantly higher muscle activities when compared with the rigid pole exercise, inducing a stronger contraction in the maximum voluntary contraction, improvement of coordination, and balance. Chung et al. ${ }^{19}$ suggested that surface electromyography of selected trunk muscles was measured with RA, EO, IO, and ES muscle in quadruped, sidebridging, and standing positions, so that flexible pole exercises are useful in the activation of trunk muscles.

The trunk strength was related to balance and function enhancement, and limb movement required. There was a significant correlation between trunk performance, balance, gait, and functional ability in patients with chronic stroke. The study of the body stabilization exercise applied to the stroke patients showed a significant improvement after the intervention, which means that the body strength exercise is related to balance and posture control. ${ }^{20}$

However, none of these studies reported a comparison of the effect of trunk muscle activation about training effect between lumbar stabilization exercise using the flexible pole and lumbar stabilization exercise using the rigid pole. Accordingly, the purpose of this study was to compare the trunk muscle activation between flexible pole and rigid pole exercise in order to evaluate whether flexible pole exercise is more effective at muscle activation in healthy adults. The second purpose of this study was to examine whether flexible pole training in accordance with the lumbar stabilization posture has the potential to be useful in the improvement of specific muscles during rehabilitation.

\section{METHODS}

\section{Subjects}

Twenty-five healthy university students were recruited for this study. The general characteristics of the subjects were as follow. The mean age, height, and weight were $20.5 \pm 1.1$ years, $167.4 \pm 0.1 \mathrm{~cm}$, and 63.8 $\pm 14.9 \mathrm{~kg}$ in the flexible pole group $(\mathrm{n}=13)$, and $20.8 \pm 1.1$ years, $168.6 \pm 0.1 \mathrm{~cm}$, and $68.8 \pm 15.6 \mathrm{~kg}$ in the rigid pole group $(\mathrm{n}=12)$, respectively.

This present study was a single blind randomized controlled trial. Participants were selected based on the selection criteria of the study. The purpose and method of research were explained in advance and all participants provided informed consent. Experiments were conducted during a 6 weeks period, and subjects were randomly assigned to one of two groups: the rigid group and the flexible pole group. None of the participants had a musculoskeletal abnormality affecting the experiment, and all participants had no a history of surgery, non-regular strength, and ongoing treatment on the shoulder. The study conformed to the standards set by the latest revision of the Declaration of Helsinki.

The sample size was analyzed using the G-power program. The effect size was 0.4 , the significance level was 0.05 , the power was $80 \%$, the number of groups was 2 , and the number of measurement was 3 . The minimum number of samples was 22 and the number of subjects included in the subject was 24 . The dropout rate was $10 \%$ and the total number of participants was 24 .

\section{Experimental method}

\section{1) Experimental procedures}

The exercise program was approximately 40 minutes, in duration per week, which consisted of 3 warm up (4 minutes), main exercise (32 minutes using flexible pole or rigid pole), and cool down (4 minutes), for a total 6 weeks. A follow-up was performed 2 weeks after the completion of the exercise program.

The flexible pole (Togu, Germany) used in this experiment is an exercise tool having weights on the both ends of an elastic bar of with a total weight of $710 \mathrm{~g}, 1,530 \mathrm{~mm}$ in length, and thickness of 9.5 
$\mathrm{mm}$ in thickness. The gripping area of $17.9 \mathrm{~cm}$ in the center part of flexible pole-allowed for a comportable grip for shaking during the exercise. The rigid pole group performed the training without the oscillation, but using a bar of the same length and weight. The flexible pole generated a vibration of 270 beats per minute $(4.6 \mathrm{~Hz})$. Voluntary arm vibration caused by the simultaneous contraction of the shoulders was transmitted to improve the strength and stability-the proprioception- around the trunk muscles.

First, The EMG was compared between curl-up and quadruped position, which performed a lot of stabilization exercises. The trunk muscles EMG activity was measured in the posture by shaking the flexible pole and the rigid pole respectively. In the posture of quadruped, non-dominant hand was shaken and measured 3 seconds later. In the posture of curl-up, both hands was shaken and measured 3 seconds later.

Second, the exercises combined with the flexible pole and rigid pole were performed in curl-up, and quadruped positions (Table 1). The exercise program was composed of eight modes, and the exercise was carried out with 30 seconds rest in between sessions. The warm- up and cool-down consisted of simple stretching exercises. The cyclic motion of the flexible pole and rigid pole was achieved by movements of the arm. We compared the electromyography (EMG) activity of trunk muscles which was normalized by percent maximal voluntary isometric contraction (\%MVIC) during the flexible pole oscillation. A rigid pole, that is non-fleixble pole, was used the length and weight that was set to the same as those of the flexible pole (Figure 1).

\section{2) Measurement method}

To measure the changes of trunk muscle activities, surface electro-

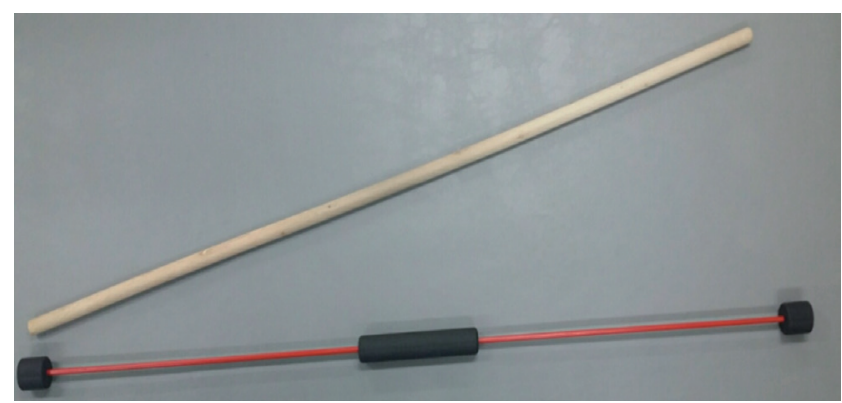

Figure 1. Flexible pole and rigid pole.

Table 1. Flexible pole exercises program

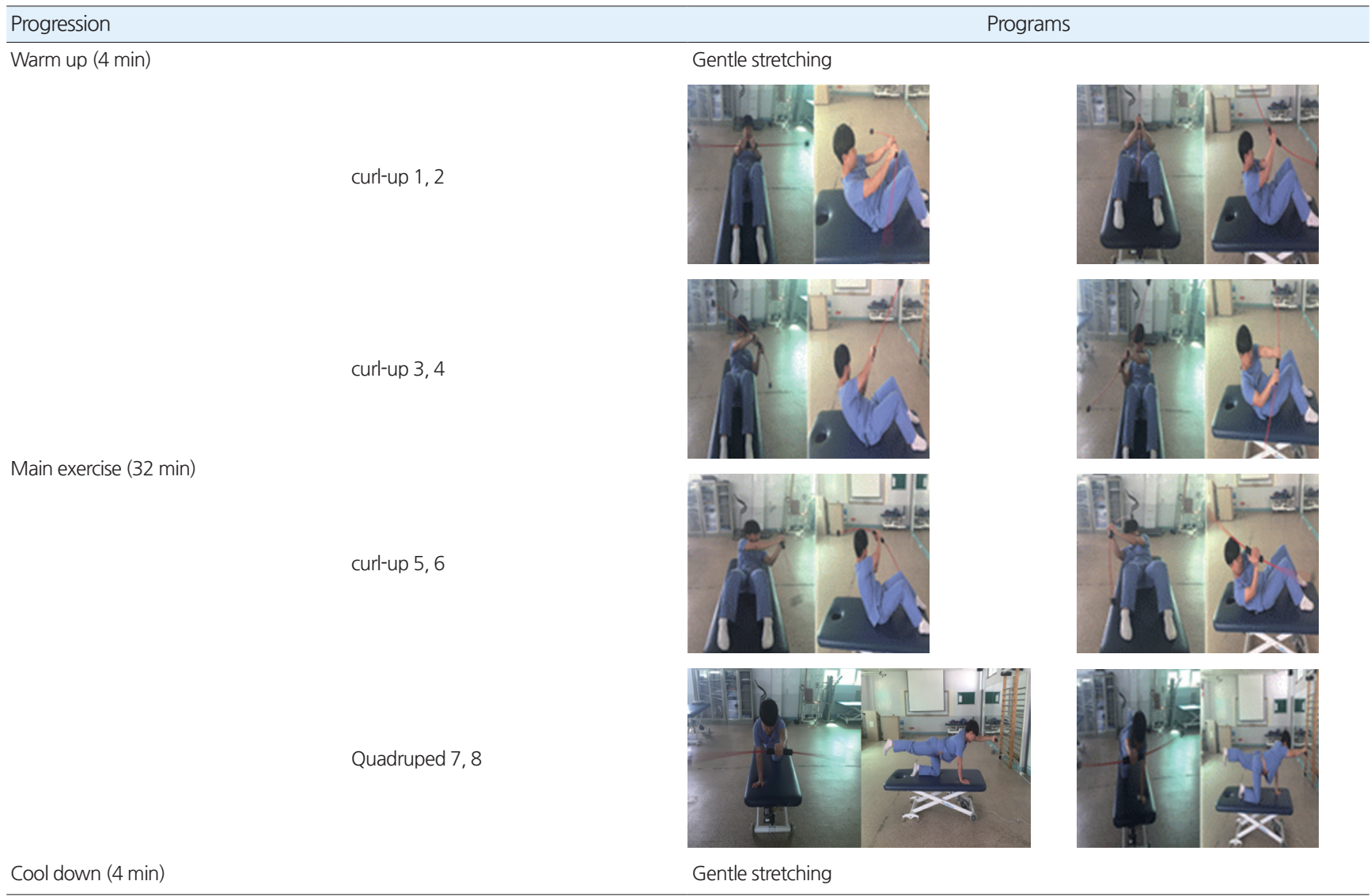


myography (EMG) (LXM 5308, Laxtha, Korea) was used. The data collected through the surface EMG signal were stored in the analysis software of Telescan (Laxtha, Korea) The EMG signal frequency was set to $1,024 \mathrm{~Hz}$, aand to enhance the accuracy, we blocked special frequencies of $60 \mathrm{~Hz}, 120 \mathrm{~Hz}$, and $180 \mathrm{~Hz}$ using a notch filter to get the accurate signal. Before attaching the electrode to minimize the resistance generated in the area of the skin removed the body hair, and the electrode was cleaned with an alcohol pad. Disposable medical electrodes were used. A ground electrode was attached to a C7 and an active electrode was attached in the rectus abdominis, external oblique, internal oblique, and erector spine. The rectus abdominis was $3 \mathrm{~cm}$ lateral to the umbilicus, external oblique was 15 $\mathrm{cm}$ lateral to the umbilicus, the internal oblique was midway between the anterior superior iliac spine and symphysis pubis, above the inguinal ligament, and erector spine was above and below the L1 level, midway of the belly muscle.

According to Danneels et al. ${ }^{21}$ the maximum voluntary isometric contraction (MVIC) values in supine and prone positions were measured in all groups. The maximum voluntary isometric contraction was measured three times per each muscle. The effective average value of 3 seconds, except one second before and after of the
5 seconds, the first and the last measured value was not used, median value was used. To measure the muscle activity during an exercise, each action was performed three times every action. The collected signals of each muscle was normalized as a percentage of MVIC on the maximum voluntary isometric contraction.

\section{Analytical method}

All data were analyzed by SPSS 21 versions for MAC. Descriptive statistics were used for the demographic and experimental data. After demonstrating the normality of the data by means of the Shapiro Wilk test, a two-way repeated measures analysis of variance was used to compare the \%MVIC of trunk muscles between the flexible pole group and the rigid pole group. Post-hoc test was conducted by using the Bonferroni method for all significant results from the twoway repeated measures ANOVA. The modified degrees of freedom values were used when the values did not satisfy Mauchly's sphericity test. The effect sizes (ES) were calculated. The following formula was used to calculate the ES: pre-post ES = posttest mean-pretest mean/ pretest standard deviation. ${ }^{22}$ The significance level was set to $\alpha=0.05$.

Table 2. Comparison of the EMG activity of trunk muscles between flexible pole and rigid pole exercises performed in quadruped and curl-up positions

(Unit: \%MVIC)

\begin{tabular}{|c|c|c|c|c|c|c|c|c|}
\hline \multirow{2}{*}{ Muscles } & \multicolumn{4}{|c|}{ Quadruped } & \multicolumn{4}{|c|}{ Curl-up } \\
\hline & Flexible pole & Rigid pole & $\mathrm{t}$ & $\mathrm{p}$ & Flexible pole & Rigid pole & $\mathrm{t}$ & $\mathrm{p}$ \\
\hline RA & $42.44 \pm 17.82$ & $34.92 \pm 14.90$ & -1.14 & 0.260 & $47.56 \pm 14.42$ & $42.06 \pm 7.65$ & -1.17 & 0.252 \\
\hline EO & $36.14 \pm 6.79$ & $30.78 \pm 11.17$ & -1.43 & 0.169 & $59.75 \pm 12.13$ & $46.33 \pm 10.36$ & -2.96 & $0.007^{*}$ \\
\hline 10 & $44.98 \pm 12.63$ & $31.89 \pm 9.39$ & -2.95 & $0.007^{*}$ & $63.01 \pm 9.98$ & $49.76 \pm 8.39$ & -3.57 & $0.002^{*}$ \\
\hline ES & $45.48 \pm 10.76$ & $33.50 \pm 11.64$ & -2.67 & $0.014^{*}$ & $23.48 \pm 5.30$ & $18.56 \pm 7.74$ & -1.86 & 0.075 \\
\hline
\end{tabular}

$\star \mathrm{p}<0.05$.

Table 3. Comparison of trunk muscle activation between the fleixbar group and rigid pole group after the flexible pole exercise

(Unit: \%MVIC)

\begin{tabular}{|c|c|c|c|c|c|c|c|c|}
\hline Muscles & Groups & Pre & After 6 wk & $\begin{array}{l}\text { Follow up } \\
(2 \mathrm{wk})\end{array}$ & & $\mathrm{F}$ & $\mathrm{p}$ & Effect size \\
\hline \multirow[t]{3}{*}{ RA } & Rigid Pole & $50.89 \pm 16.51$ & $65.62 \pm 25.10$ & $57.92 \pm 14.56$ & time & 9.244 & $0.000^{*}$ & 0.69 \\
\hline & & & & & group & 1.258 & 0.274 & \\
\hline & Flexible Pole & $52.98 \pm 16.38$ & $77.22 \pm 24.42$ & $62.68 \pm 13.40$ & time*group & 0.109 & 0.744 & 1.16 \\
\hline \multirow[t]{3}{*}{ EO } & Rigid Pole & $51.73 \pm 8.01$ & $66.88 \pm 21.60$ & $61.55 \pm 18.02$ & time & 13.652 & $0.000^{*}$ & 0.93 \\
\hline & & & & & group & 0.402 & 0.532 & \\
\hline & Flexible Pole & $54.68 \pm 13.07$ & $72.28 \pm 15.21$ & $62.83 \pm 14.32$ & time*group & 0.218 & 0.805 & 1.24 \\
\hline \multirow[t]{3}{*}{10} & Rigid Pole & $52.46 \pm 6.22$ & $63.93 \pm 14.53$ & $62.97 \pm 15.55$ & time & 5.454 & $0.007^{*}$ & 1.02 \\
\hline & & & & & group & 0.424 & 0.521 & \\
\hline & Flexible Pole & $56.45 \pm 16.21$ & $66.29 \pm 16.50$ & $64.81 \pm 15.54$ & time*group & 0.050 & 0.932 & 0.60 \\
\hline \multirow[t]{3}{*}{ ES } & Rigid Pole & $54.47 \pm 6.97$ & $65.61 \pm 13.58$ & $58.91 \pm 7.40$ & time & 6.485 & $0.012^{*}$ & 1.03 \\
\hline & & & & & group & 0.286 & 0.598 & \\
\hline & Flexible Pole & $54.68 \pm 8.58$ & $69.75 \pm 13.35$ & $62.34 \pm 12.57$ & time*group & 0.165 & 0.738 & 1.34 \\
\hline
\end{tabular}

$* p<0.05$. 


\section{RESULTS}

There was a significant difference with regarding the trunk muscle activation between the internal oblique and erector spine muscles in the exercises performed in the quadruped posture $(\mathrm{p}<0.05)$. Moreover, there was a significant difference with respect to trunk muscle activation between the external oblique and internal oblique muscles in the exercises performed in the curl-up position $(\mathrm{p}<0.05)$ (Table 2).

As a result, interaction of time and groups of all trunk muscles was not significant. As the time yields significant main effects, a repeated measures analysis of variance was performed for time. Posthoc analysis demonstrated a significant increase in the post-exercise values, as compared with the pre-exercise values $(\mathrm{p}<0.05)$. The \%MVIC values for the RA, EO, IO, and ES muscles were significantly greater in 6 weeks, follow-up when comparing pre-test in all group $(\mathrm{p}<0.05)$. There was no significant difference between the values for flexible pole and rigid pole. The flexible pole group showed a much higher effect size than the rigid pole group after the intervention in RA (1.16, 68\% increase), EO (1.24, 33\% increase), and ES (1.34, 30\% increase)(Table 3).

\section{DISCUSSION}

The aim of this study was to determine if the flexible pole combined with lumbar stabilization depending on vibration could affect trunk muscle activation (RA, EO, IO, ES) after intervention and to investigate whether the effect of general lumbar stabilizing exercise with fleixbar on muscle activities. EMG was used to investigate the flexible pole effect. EMG is a biological activity of the movement made by the existing motor unit generated during muscle contraction. It is utilized as the most common method in the analysis of biomechanics and motor control. ${ }^{23}$

In this study, the flexible pole combined with an exercise in curlup and quadruped positions commonly induced a greater activation in the internal oblique than an exercise in curl-up and quadruped positions without the use of flexible pole. This means that a stimulation of continuous vibration provided by the flexible pole induces spine stabilizing muscle. The IO muscle is suggested the key muscle that maintains for stabilized lumbar spine, because this muscle is inserted into the thoraco-lumbar fascia, on all lumbar vertebral bodies. Moreover, IO muscles are situated deeper within the body than EO muscles. The IO muscles activated by the flexible pole attribute to the feed-forward mechanisms. Delayed feed-forward activation is caused by lumbar problems. ${ }^{24}$ These results were consistent with a previous study that showed that quadruped, standing, and side-bridging positions with a fleixbar was associated with higher IO muscles activation. ${ }^{19}$ Goncalves et al. ${ }^{25}$ reported that the IO muscles presented a $72 \%$ greater muscle activity during the exercises performed with a flexible pole than with a non-flexible pole.

The curl-up exercise with a flexible pole showed a significantly higher IO and EO muscles activation than the curl-up exercise with a rigid pole. Trunk rotator included $\mathrm{IO}$ and $\mathrm{EO}$ muscles are needed for trunk stability. The curl-up exercise using flexible pole is more effective for trunk stability than the curl-up exercise using rigid pole. This is thought to induce greater trunk rotation since shaking occurs on a horizontal plane, while the flexibar is held in a vertical orientation. Anders et al. ${ }^{26}$ argued that could different from muscle activity due to the oscillation direction and plane. The repetition of shaking horizontally while holding the pole vertically was dependent on how one shakes the flexible pole. ${ }^{27}$ The curl-up exercise combined with a fleixbar showed a significant improvement in the IO and EO muscles activation. ${ }^{28}$ Sánchez-Zuriaga et al. ${ }^{27}$ reported that the vertical orientation on the horizontal plane produced the greatest activation levels of the internal oblique and external oblique muscles. Muscle activation from using an oscillating pole were not affected by posture. Mileva et al. ${ }^{15}$ reported that the amplitude of electromyography was significantly higher during an exercise using flexible pole than shambar exercise in a 1-leg squat position in healthy adults. In this study, the ES muscle showed a significant difference in quadruped position between the use of flexible pole and rigid pole. This result can be explained through the equilibrium reaction against the continuous vibration stimulus. ${ }^{28}$

The trunk muscles activation of both groups was significantly higher after 6 weeks of intervention than pre-test. Especially, RA, $\mathrm{IO}$, and ES muscles were maintained throughout the follow-up, except for EO muscle. This suggests that the flexible pole exercise transmitted $5 \mathrm{~Hz}$ vibration in the arm muscles, which was effectively carried from the hand to the arm and trunk muscles. The vibrations transmitted via flexible pole produced cyclical sway, demanding trunk muscle activation to stabilize the body. ${ }^{25}$ Vibration transmitted to the belly muscle or tendon by the flexible pole can induce tonic vibration reflex (TVR), which is a sustained contraction of a 
muscle subjected to vibration. ${ }^{13}$ This reflex is caused by a vibratory activation of the muscle spindles. ${ }^{29}$

There was no change in the trunk muscle activation between the flexible pole and rigid pole, which implies that a stimulus from the flexible pole alone was not strong enough to result in significant changes in trunk muscles activation among healthy adults muscles. Also, it was considered becasue of body instability, instability of force transfer process, and pelvic dynamics and body alignment.

However, the cause of no difference between the groups may be due to the stable support of the surface during the movement. It would not be applied in a more unstable posture. ${ }^{30}$ It seems that the trunk muscle activation was dependent on surface stability. The exercises increased the swaying movement of the trunk when the body's center of mass was on an unstable surface. When performing the quadruped and curl-up exercises, the activity level of trunk muscles on an unstable surface was greater than that on a stable surface. Exercise postures were performed on a stable surface in our study. It seems that to recruit the activation of as many trunk muscles as possible, unstable posture is necessary. It was previously reported that vibration applied during an unstable squat produced significantly greater muscle EMG amplitudes than during an stable squat. ${ }^{31}$ Vera-Garcia et al. ${ }^{32}$ reported that there was a greater activation in the rectus abdominis and external oblique by the curl-up exercise on an unstable surface than on a stable surface. Although both groups did not show a significant difference, a comparison of the effect size between the two groups showed that the flexible pole group had higher RA, IO, and ES than the rigid pole group. In order to recruit as many trunk muscles, flexible pole combined with lumbar stabilization may be helpful in improving muscle strength.

A limitation of the present study is the small number of sample size, as it resulted in generalizability difficulties. Although the flexible pole initiates activity-induced vibrations, all subjects did not control the amplitudes of vibration that may affect the trunk muscles activation. This study did not measure the muscle activity in various general trunk exercises. The investigation was performed with healthy adults. Therefore, the question still remains un answered whether the trunk muscles activation can still be evoked in patients with low back pain. Future studies will be needed to further investigate if there are any differences in each posture for people with trunk instability.

\section{REFERENCE}

1. Panjabi MM. The stabilizing system of the spine. Part i. Function, dysfunction, adaptation, and enhancement. J Spinal Disord. 1992;5(4):3839 .

2. Gardner-Morse MG, Stokes IA. The effects of abdominal muscle coactivation on lumbar spine stability. Spine. 1998;23(1):86-91.

3. McGill SM, Grenier S, Kavcic N et al. Coordination of muscle activity to assure stability of the lumbar spine. J Electromyogr Kinesiol. 2003; 13(4):353-9.

4. Bergmark A. Stability of the lumbar spine. A study in mechanical engineering. Acta Orthop Scand Suppl. 1989;230:1-54.

5. Koumantakis GA, Watson PJ, Oldham JA. Trunk muscle stabilization training plus general exercise versus general exercise only: randomized controlled trial of patients with recurrent low back pain. Phys Ther. 2005;85(3):209-25.

6. Scott IR, Vaughan AR, Hall J. Swiss ball enhances lumbar multifidus activity in chronic low back pain. Phys Ther Sport. 2015;16(1):40-4 .

7. Shamsi MB, Rezaei M, Zamanlou M et al. Does core stability exercise improve lumbopelvic stability (through endurance tests) more than general exercise in chronic low back pain? A quasi-randomized controlled trial. Physiother Theory Pract. 2016;32(3):171-8.

8. Moraes AC, Pinto RS, Valamatos MJ et al. EMG activation of abdominal muscles in the crunch exercise performed with different external loads. Phys Ther Sport. 2009;10(2):57-62.

9. Rossi DM, Morcelli MH, Marques NR et al. Antagonist coactivation of trunk stabilizer muscles during pilates exercises. J Bodyw Mov Ther. 2014;18(1):34-41.

10. Hansen FR, Bendix T, Skov P et al. Intensive, dynamic back-muscle exercises, conventional physiotherapy, or placebo-control treatment of low-back pain. A randomized, observer-blind trial. Spine. 1993;18(1): 98-108.

11. Lee SJ, Kim YN, Lee DK. The effect of flexi-bar exercise with vibration on trunk muscle thickness and balance in university students in their twenties. J Phys Ther Sci. 2016;28(4):1298-302.

12. Ruzene JR, Morcelli MH, Navega MT. Equilibrium in women with osteoporosis submitted to balance training with and without an oscillating vibratory pole. J Bodyw Mov Ther. 2016;20(1):35-41.

13. Cardinale M, Rittweger J. Vibration exercise makes your muscles and bones stronger: fact or fiction? J Br Menopause Soc. 2006;12(1):12-8.

14. Issurin VB, Tenenbaum G. Acute and residual effects of vibratory stimulation on explosive strength in elite and amateur athletes. J Sports Sci. 1999;17(3):177-82.

15. Mileva KN, Kadr M, Amin N et al. Acute effects of flexi-bar vs. Shambar exercise on muscle electromyography activity and performance. J Strength Cond Res. 2010;24(3):737-48.

16. Cardinale M, Bosco C. The use of vibration as an exercise intervention. Exerc Sport Sci Rev. 2003;31(1):3-7.

17. Buteau JL, Eriksrud O, Hasson SM. Rehabilitation of a glenohumeral instability utilizing the body blade. Physiother Theory Pract. 2007;23(6): 333-49.

18. Lister JL, Del Rossi G, Ma F et al. Scapular stabilizer activity during bodyblade, cuff weights, and thera-band use. J Sport Rehabil. 2007; 16(1):50-67. 
19. Chung JS, Park S, Kim J et al. Effects of flexi-bar and non-flexi-bar exercises on trunk muscles activity in different postures in healthy adults. J Phys Ther Sci. 2015;27(7):2275-8.

20. Verheyden G, Vereeck L, Truijen S et al. Trunk performance after stroke and the relationship with balance, gait and functional ability. Clin Rehabil. 2006;20(5):451-8.

21. Danneels LA, Cagnie BJ, Cools AM et al. Intra-operator and inter-operator reliability of surface electromyography in the clinical evaluation of back muscles. Man Ther. 2001;6(3):145-53.

22. Cohen J. Things I have learned (so far). American psychologist. 1990; 45(12): 1304

23. Ferrante $S$, Pedrocchi A, Ianno $M$ et al. Functional electrical stimulation controlled by artificial neural networks: pilot experiments with simple movements are promising for rehabilitation applications. Funct Neurol. 2004;19(4):243-52.

24. Hodges PW, Richardson CA. Inefficient muscular stabilization of the lumbar spine associated with low back pain. A motor control evaluation of transversus abdominis. Spine. 1996;21(22):2640-50.

25. Goncalves M, Marques NR, Hallal CZ et al. Electromyographic activity of trunk muscles during exercises with flexible and non-flexible poles. J Back Musculoskelet Rehabil. 2011;24(4):209-14.

26. Anders C, Wenzel B, Scholle HC. Activation characteristics of trunk muscles during cyclic upper-body perturbations caused by an oscillating pole. Arch Phys Med Rehabil. 2008;89(7):1314-22.

27. Sánchez-Zuriaga D, Vera-Garcia FJ, Moreside JM et al. Trunk muscle activation patterns and spine kinematics when using an oscillating blade: influence of different postures and blade orientations. Arch Phys Med Rehabil. 2009;90(6):1055-60.

28. Kim JH, So KH, Bae YR et al. A comparison of flexi-bar and general lumbar stabilizing exercise effects on muscle activity and fatigue. J Phys Ther Sci. 2014;26(2):229-33.

29. Martin BJ, Park HS. Analysis of the tonic vibration reflex: influence of vibration variables on motor unit synchronization and fatigue. Eur J Appl Physiol Occup Physiol. 1997;75(6):504-11.

30. Imai A, Kaneoka K, Okubo Y et al. Trunk muscle activity during lumbar stabilization exercises on both a stable and unstable surface. J Orthop Sports Phys Ther. 2010;40(6):369-75.

31. Roelants M, Verschueren SM, Delecluse C et al. Whole-body-vibrationinduced increase in leg muscle activity during different squat exercises. J Strength Cond Res. 2006;20(1):124-9.

32. Vera-Garcia FJ, Grenier SG, McGill SM. Abdominal muscle response during curl-ups on both stable and labile surfaces. Phys Ther. 2000; 80(6):564-9. 